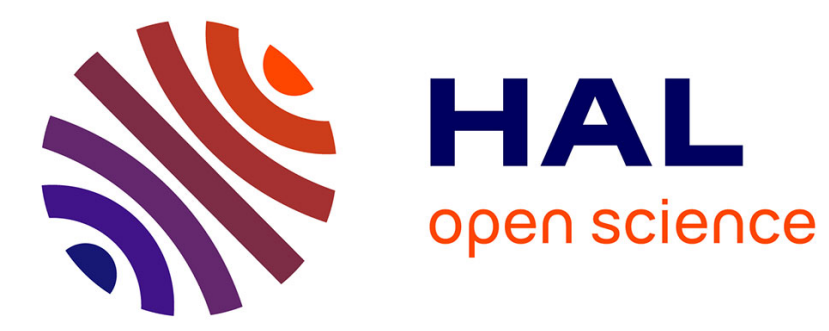

\title{
La pénurie des ressources européennes en bois de placage \\ Paul Silvy-Leligois
}

\section{To cite this version:}

Paul Silvy-Leligois. La pénurie des ressources européennes en bois de placage. Revue forestière française, 1954, 8-9, pp.501-510. 10.4267/2042/27013 . hal-03384166

\section{HAL Id: hal-03384166 \\ https://hal.science/hal-03384166}

Submitted on 18 Oct 2021

HAL is a multi-disciplinary open access archive for the deposit and dissemination of scientific research documents, whether they are published or not. The documents may come from teaching and research institutions in France or abroad, or from public or private research centers.
L'archive ouverte pluridisciplinaire HAL, est destinée au dépôt et à la diffusion de documents scientifiques de niveau recherche, publiés ou non, émanant des établissements d'enseignement et de recherche français ou étrangers, des laboratoires publics ou privés. 


\section{REVUE FORESTIERE FRANÇAISE}

I954

Septembre

No $8-9$

Ians ce numéro: P. Silvy-Leligois: La pénurie des ressources européennes en bois de chêne de placage. - R. VinEy : La production des chênes de tranchage peut-elle être améliorée par les aménagements? - J. Boutin: Evolution des techniques de construction de routes. - G. Roger: Réalisation d'une route en sol stabilisé. Route forestière de La Table.

\section{A NOS LECTEURS}

Au début du mois d'aôit, un accident mettait cruellement en deuil notre imprimeur-éditeur, en même temps qu'il le blessait grièvement.

Madame Thomas collaborait activement à l'édition de notre Revue. Nous lui devons une vive reconnaissance et son absence se fera gravement ressentir.

Nous prions Monsieur Thomas de vouloir bien agréer, de la part de tous nos collaborateurs, l'expression de nos condoléances très émues, en même temps que nos souhaits de prompt rétablissement.

Le Comité de Rédaction.

\section{LA PÉNURIE DES RESSOURCES EUROPEEENNES EN BOIS DE CHÊNES DE PLACAGE}

Le Congrès National du Bois I953-54 a, une fois de plus, souligné l'intérêt du placage comme matériau moderne. Il a fait ressortir l'augmentation de consommation de matière première due à la fois à la variété d'emploi des contreplaqués, et à l'appétit, de plus en plus grand, des machines à trancher ou à dérouler. I1 s'engage, entre les usines d'utilisation et la forêt productrice, une véritable lutte de vitesse qui peut placer les économistes devant un problème sans issue, si des solutions de rechange ne sont pas étudiées à l'avance.

Au pessimisme des uns s'oppose la confiance de certains qui voient, dans les essences de nos territoires d'outre-mer, la matière première inépuisable et à portée de la main. Le Congrès de Strasbourg a, sur ce point encore, donné des précisions. Toutes les essences africaines ne sont pas propres à la fabrication des contreplaqués: bois trop tendre ou trop dur, de mauvaise conservation, en trop faible . 
quantité, etc... Parmi ceux qui répondent aux critères d'utilisation pour le placage, il n'en existe qu'un petit nombre susceptible de remplacer nos bois durs indigènes, notamment le noyer et le chêne, qui fournissent toujours les placages riches pour l'ébénisterie et la décoration. Les prix qu'atteignent les grumes de ces deux essences vont sans cesse en augmentant et prouvent la préférence marquée des utilisateurs européens pour ces bois et la concurrence qu'ils font jouer pour s'assurer un approvisionnement suffisant: si, en France, certains arbres de Tronçais ont atteint le prix de $150000 \mathrm{~F}$ au mètre cube, que dire des chênes d'Outre-Rhin qui trouvent preneur à 3500 D.M., même s'il s'agit du mètre cube abattu et façonné à bord de route! Les chiffres cités se rapportent à deux pays encore producteurs de gros chênes de placage; or, il existe, en Europe, de nombreux pays importateurs de ces bois et qui n'en produisent pas sur leur sol.

En limitant cet article à l'inventaire des ressources européennes en gros chênes propres au placage, nous ne prétendons pas en fournir une statistique exacte et définitive, mais donner seulement un aperçu des zones traditionnelles d'approvisionnement et de leurs possibilités pour le présent et dans l'avenir.

Une remarque s'impose d'abord: il pourra sembler, au cours de cet exposé, que les pays producteurs ont fait preuve d'imprévoyance, en dilapidant, pour des emplois vulgaires, des richesses en bois qui mériteraient une destination plus noble. Mais la confrontation des dates prouve que les grosses exploitations ont de beaucoup précédé l'essor de l'industrie du placage dans les pays correspondants et qu'il ne s'agit plus, actuellement, que de liquidation de restes de beaux peuplements, qui n'avaient d'ailleurs pas été destinés à cet usage. Nous avons déjà souligné l'incertitude des prévisions en matière forestière; nous n'y reviendrons pas.

Nous envisagerons successivement les trois pays d'Europe qui ont .eu, depuis un siècle, à leur disposition, de gros volumes de chênes de fort diamètre, puis nous établirons un bilan rapide des ressources actuelles de l'Europe en grumes de chênes de placage. laissant à de plus qualifiés le soin de proposer des situations propres à assurer une relative continuité dans l'approvisionnement des industries d'utilisation.

\section{I. - Yougoslavie.}

Il s'agit sans doute de la plus grande étendue de chênaies qui ait existé au $\mathrm{XIX}^{\boldsymbol{\theta}}$ siècle en Europe. Situés dans les fonds et sur les coteaux qui avoisinent les vallées de la Save et de la Drave, ces peuplements, en majorité de chêne pédonculé, occupaient encore plus de Ioo ooo hectares il y a un siècle. Les portions les plus âgées semblent avoir fait. entre I850 et I900, l'objet d'exploitations abusives par enlèvement de quantités prodigieuses de gros chênes de I $\mathrm{m}$ à $2 \mathrm{~m}$ de diamètre, plus spécialement pour la fabrication des 
LA PÉNURIE EN CHÊNES DE PLACAGE

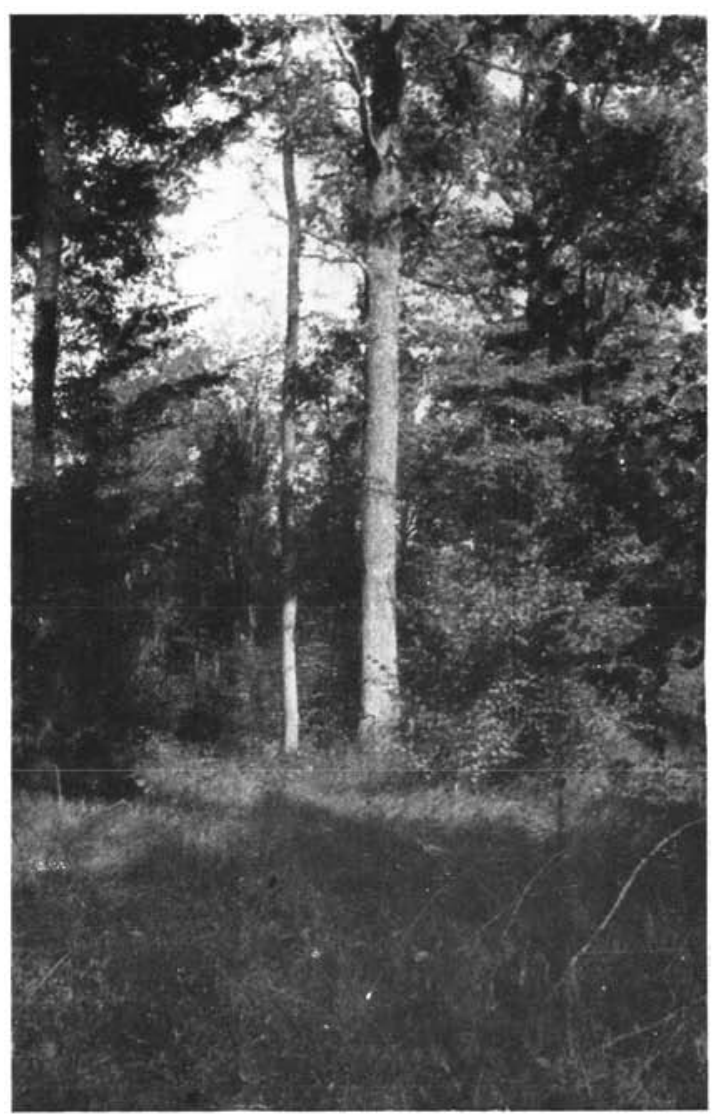

Yougoslavie

Sud de Zagreb

Peuplement clairiéré de

vieux chènes pédonculés

et recru d'âges variés.

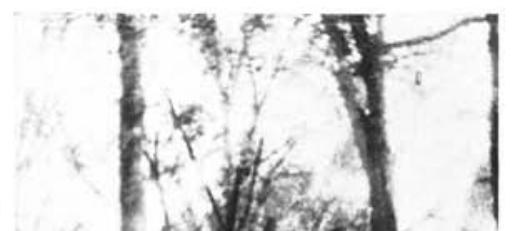

Yougoslavie

Forêt de Banor-Dol

Chênes pédonculés en massif complet sans sous-étage.

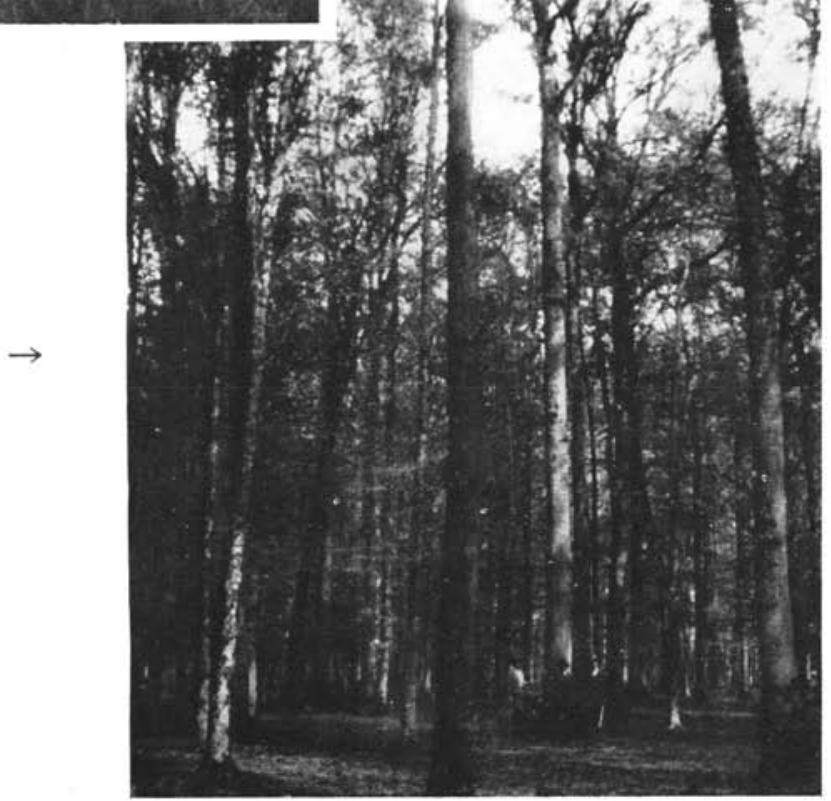

(Clichés Pfrrin.) 
LA PÉNURIE EN CHÊNES DE PLACAGE

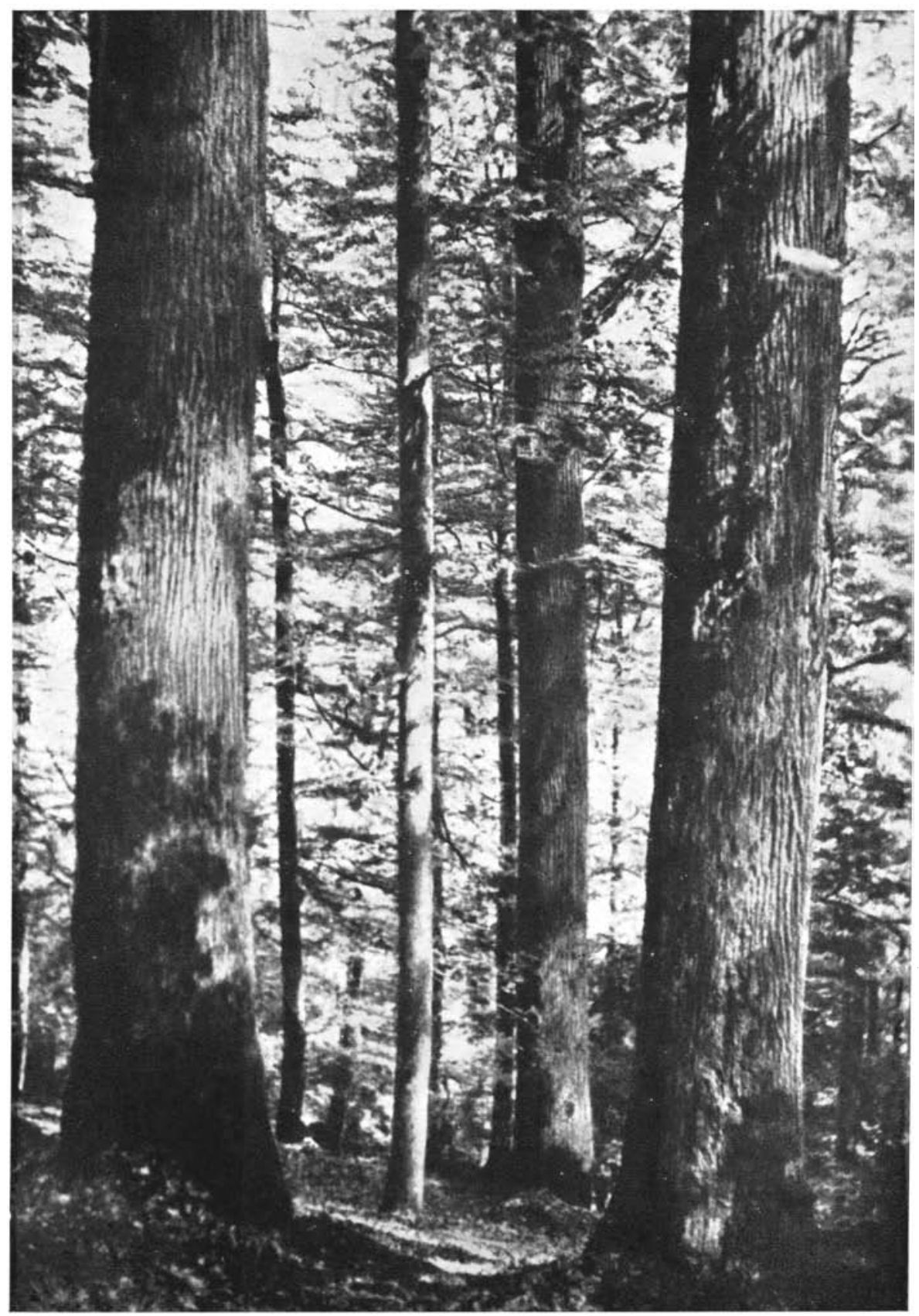

Allemagne

Forêt du Spessart

(District de Rothenbuch)

Peuplement de vieux chênes rouvres en massif complet sans sous-étage.

(Cliché Zhum.)

Extrait de Intersylva, juillet $195^{2}$. 
merrains. Depuis le début du siècle, les chênes de Slavonie ont fait prime sur le marché européen et donné lieu à un commerce important d'exportation.

Nous manquons de chiffres récents pour fixer les volumes exploitables et les moyennes annuelles de réalisation. Pour le passé, une expertise faite en 1908 par Huffel, dans les domaines du Prince de Schaumburg-Lippe indiquait que les 9/Io des peuplements avaient été exploités abusivement durant la seconde moitié du XIX ${ }^{\mathrm{e}}$ siècle.

Poskin révèle qu'en 1920 , il restait seulement 7000 ha de vieux peuplements de chênes âgés de plus de 200 ans, mais que cette surface avait subi une forte diminution depuis 1920.

Le chiffre de 7000 ha correspond bien à la proportion notée en I926 par Manoylovitch et qui s'élevait à 5,9\% de la surface totale, en bois de I40 ans et plus.

Enfin, Perrin précise' qu'en I922, il reste quelques milliers d'hectares de vieux peuplements, et qu'après leur réalisation, il y aura un vide de 40 ans, si même les peuplements actuellement en croissance acquièrent des dimensions suffisantes et des qualités équivalentes.

En ce qui concerne la consommation, les besoins de l'industrie du placage étaient jusqu'en I939, largement couverts par les ressources nationales, puisque ce pays exportait des grumes de belle qualité. L'industrie encore peu développée absorbait certainement de 8 à $10.000 \mathrm{~m}^{3}$ de grumes. Depuis 1945 , l'essor industriel a sans doute augmenté cette consommation, alors que les ressources allaient en diminuant.

\section{II. - Allemagne.}

Hessmer classe, parmi les régions renfermant une proportion appréciable de chênes âgés de plus de Ioo ans:

- la Rhénanie (Cassel, Wiesbaden et Coblence) avec I7 000 ha

- la Franconie inférieure .............. 8000 ha

- la Sarre et le Palatinat .............. 7000 ha

- le Bade ..................... I I ooo ha

La plupart de ces régions semblent posséder des chênes rouvres de dimensions et de qualité suffisantes pour fournir des bois de tranchage; mais la Sarre et le Palatinat (sporadiquement) et le Spessart (en massif complet) représentent les centres les plus importants de production de gros chênes.

Dans le premier subsistent quelques restes de vieux peuplements, dont les chênes, de grande qualité, sont très recherchés par les usines de placage voisines. Ils n'apparaissent qu'en petits îlots au milieu de peuplements plus jeunes, et ne fournissent annuellement qu'un millier de mètres cubes de gros bois. Mais ils sont une indication précieuse pour l'avenir et prouvent qu'avec une sylviculture 
appropriée, ces régions sont susceptibles de produire des chênes. de qualité exceptionnelle.

Par opposition avec ce premier centre, oì les ressources se trouvent disséminées, le Spessart représente un massif tout à fait unique en Allemagne. Situé à $80 \mathrm{~km}$ à l'Ouest de Mayence, près de la ville d'Aschaffenbourg, il englobe une superficie boisée de 50000 hectares, dont moitié appartient à l'Etat, et moitié aux communes et aux particuliers.

Au centre de ce massif se trouve un ensemble de 5500 hectares dont les bois ont une valeur exceptionnelle.

Nous ne pouvons donner que des chiffres approximatifs sur les volumes restant actuellement sur pied. Il y a lieu de distinguer les peuplements clairiérés de 400 à 500 ans, et les peuplements pleins de 200 à 300 ans.

Parmi les premiers, Huffel cite ces deux chiffres:

- en I837, il existait 5000 ha de peuplements clairs de chènes de. 400 à 500 ans;

- en I897, il existait 600 ha des mêmes peuplements représentant un volume global de $260000 \mathrm{~m}^{3}$.

En reprenant les données fournies par le Parc Rohrberg, avec un matériel de $300 \mathrm{~m}^{3}$ à l'hectare, il y aurait eu, en $\mathrm{I} 837$, I 500000 $\mathrm{m}^{3}$; et la coupe annuelle de 1837 à $\mathrm{I} 897$ aurait représenté environ $20000 \mathrm{~m}^{3}$.

Pour. les seconds, Huffel dit encore qu'en I908, les massifs pleins couvraient 850 ha; avec un matériel moyen de $400 \mathrm{~m}^{3}$, ces peuplements auraient renfermé $340000 \mathrm{~m}^{3}$.

ENDRES leur attribue, en I929, une surface de 500 hectares et fait état pour l'ensemble des vieux chênes du Spessart d'un volume de $624000 \mathrm{~m}^{3}$ dont:

$212000 \mathrm{~m}^{3}$ pour les vieux chênes isolés,

4I $2000 \mathrm{~m}^{3}$ pour les vieux chênes en massifs pleins.

Le guide du Congrès de I935 attribue à ces mêmes peuplements en massifs une surface de $45^{\circ}$ ha; et ORTH, en I942, évalue à $400000 \mathrm{~m}^{3}$ le volume total des vieux chênes du Spessart.

La coupe annuelle de I929 à I942 aurait donc représenté au maximum I7 $000 \mathrm{~m}^{3}$, chiffre très voisin de celui cité par HuFfeL qui parlait en 1908 d'une réalisation annuelle de $19000 \mathrm{~m}^{3}$.

En réalité, le volume de bois d'œuvre chêne exploité semble s'établir aux environs de $\mathrm{I} 2000 \mathrm{~m}^{3}$ par an.

Le guide dı Congrès cite les chiffres de:

$4000 \mathrm{~m}^{3}$ pour la coupe annuelle dans le district de Rohrbrunn (580o ha),

$5000 \mathrm{~m}^{3}$ pour la coupe annuelle dans le district de Rimpar (4 200 ha).

Enfin, Köstler, exposant la récolte des bois d'œuvre dans l'ensemble du Spessart évalue en 1936 à $\mathrm{I} 2000 \mathrm{~m}^{3}$ le bois d'œuvre 
thêne et à $3000 \mathrm{~m}^{3}$ le volume de chênes de tranchage exploités annuellement.

Si ce chiffre de $12000 \mathrm{~m}^{3}$ n'a pas été dépassé durant la période . 1936-1946, et nous en doutons, le volume des gros chênes est tombé cependant de 400000 à $280000 \mathrm{~m}^{3}$, dont moins d'un quart susceptible de fournir des bois de placage. Et, au rythme actuel, en moins de 30 ans, ces réserves seront totalement épuisées.

En ce qui concerne la consommation, l'approvisionnement en bois indigène était nettement insuffisant. En effet, en dehors du Spessart, qui pouvait fournir annuellement $4000 \mathrm{~m}^{3}$, un apport de $2000 \mathrm{~m}^{3}$ au. maximum pouvait provenir des autres régions déjà citées (Palatinat, Sarre, Rhénanie, Bade) et de certains cantons souvent décrits comme riches en vieux chênes, Freienland, par exemple.

En regard de ces ressources intérieures s'élevant à $6000 \mathrm{~m}^{3}$, l'industrie allemande devait atteindre en I928 une production annuelle de placages de chêne de $5000 \mathrm{~T}$; ce qui représente environ $16000 \mathrm{~m}^{3}$ de grumes.

Où l'Allemagne pouvait-elle trouver la matière première nécessaire?

En Autriche, dont la production en grumes de placage atteignait en 1935: i $600 \mathrm{~m}^{3}$;

En Pologne, dont certains grands massifs (Bialowiecza, par exemple) fournissaient annuellement $6000 \mathrm{~m}^{3}$ de chênes de placage;

En Yougoslavie, qui, en 1938, exportait I4 $845 \mathrm{~T}$ de grumes de chênes, soit environ $20000 \mathrm{~m}^{3}$, pour un prix de $\mathrm{r} 4576000$ dinars. Ce qui fait ressortir le prix du mètre cube moyen à 725 dinars. Or, les prix de 1938 sont restés approximativement ceux pratiqués de I929 à I93I; à cette époque, le mètre cube sur wagon départ se vendait 3500 dinars pour la bille de pied et 300 dinars pour la dernière tronce. Sur ces bases, le prix de 725 dinars indiquerait, pour l'ensemble des pays acheteurs, une proportion de qualités supérieures égale à $2600 \mathrm{~m}^{3}$ contre $\mathrm{I} 7400 \mathrm{~m}^{3}$ de qualités moyennes. Or, l'Allemagne est citée comme le pays importateur ayant acheté les bois les plus précieux et payé les meilleurs prix. Sur les $20000 \mathrm{~m}^{3}$ exportés, nous pouvons évaluer à $3000 \mathrm{~m}^{3}$ au moins le volume de grumes de placage que l'Allemagne pouvait trouver en Yougoslavie.

Enfin, la France fournissait certainement à l'industrie allemande un appoint appréciable, difficile à chiffrer avant guerre, faute de statistiques douanières. Mais, de $\mathrm{I} 94 \mathrm{I}$ à $\mathrm{I} 944,2000 \mathrm{~m}^{3}$ ont été exportés chaque année, soit directement, soit par la Suisse.

En totalisant toutes ces ressources, qui ont pu se combiner jusqu'en I944, la consommation de l'industrie allemande en grumes de chênes de placage s'élèverait à $18000 \mathrm{~m}^{3}$, chiffre très voisin de celui indiqué pour I928.

Mais ce chiffre est certainement dépassé actuellement. Nous 
n'avons aucune raison de penser que la consommation de gros chênes ait diminué, alors que la production en contreplaqués pour la seule zone occidentale atteint en I950, le volume produit par toute l'Allemagne en I936-37.

\section{III. - France.}

Les grandes régions productrices de bois de chênes de placage sont le centre et l'ouest. Du moins faut-il préciser que les forêts considérées traditionnellement comme productrices de tels bois sont concentrées dans ces régions. Mais il existe, en France, de nombreux massifs non spécialisés, dans lesquels se trouvent, en plus ou moins grande quantité, des bois répondant à ces spécifications. Ils n'ont pas jusqu'ici fait l'objet d'inventaires précis, ni d'aménagement en ce qui concerne les délais de réalisation de ces bois. Leur apport n'est cependant pas négligeable.

Pour ne parler que des massifs spécialisés, nous les rangerons en trois groupes, d'une part: l'Allier, Indre, Cher; d'autre part: Loir-et-Cher et Indre-et-Loire; enfin Orne et Sarthe.

\section{I) Groupe Allier - Indre - Cher.}

Les forêts de la grande Maitrise du Bourbonnais ont fait l'objet de nombreuses études. Leur histoire reste liée à l'œuvre des Commissaires-Réformateurs, dont l'influence s'est exercée surtout entre I670 et I778. A partir de I778, jusqu'à l'application des doctrines françaises d'aménagement en futaie régulière par affectations, c'està-dire jusqu'à 1835 , ces forêts ont subi de nombreuses vicissitudes : aliénations, retour au taillis pour les besoins des forges, etc...

Les plus vieux arbres nés à la suite de l'application des Réformations auraient donc au maximum:

et au minimum:

$$
1954-\mathrm{I} 670=284 \text { ans }
$$

$$
\mathrm{I} 954-\mathrm{I} 778=\mathrm{I} 76 \text { ans }
$$

En réalité, il n'existe pratiquement pas de peuplements de I76 à 250 ans, car les exploitations des forges ont été faites souvent, entre 1778 et 1800 , aux dépens de perchis qui ont été ramenés à l'état de taillis et dont l'exploitation n'a été suivie ni de repeuplements, ni de mise en vieillissement. Ces prescriptions ont, par contre, été appliquées à partir de 1800 , et les concessions aux maitres de forges, à charge de repeuplements, ont donné de beaux cantons, d'origine artificielle, ou des perchis sur souche, âgés aujourd'hui de $\mathrm{I} 5 \mathrm{O}$ à $\mathrm{I} 75$ ans.

Que reste-t-il des pluș vieux peuplements ?

En forêt de Tronçais, l'inventaire de 1952 révèle:

ı68 ha de peuplements âgés de plus de 250 ans (y compris 79 ha de Réserve artistique), 
o ha de peuplements âgés de 176 à 250 ans,

I 479 ha de peuplements âgés de I 50 à I 76 ans, dont la dimension n'est pas suffisante pour y trouver des bois de tranchage $(0,45 \mathrm{~m}$ de diamètre moyen à I,30 $\mathrm{m})$.

Mise à part la Réserve artistique, il reste à exploiter, durant les 50 ans à venir, quelque $15000 \mathrm{~m}^{3}$ des gros chênes qui ont fait la réputation de Tronçais et dont la raréfaction explique la faveur actuelle.

En forêt de Moladier, les vestiges du Trésor ne sont plus représentés que par quelques arbres: en forêt de Bagnolet, les derniers peuplements renfermant de gros chênes ont été exploités en 1942. Dans ces deux forêts, dont la qualité était également réputée, il faudra attendre une trentaine d'années pour recruter de nouveau des chênes de $0,60 \mathrm{~m}$ et plus.

Les forêts de Dreuille, Civrais, Soulongis, Boisplan, etc..., renferment de jeunes futaies pleines de promesses, mais de trop faibles dimensions pour approvisionner le marché des placages.

Dans le Cher et l'Indre, il existe des poussières de massifs dont certains cantons fournissent des bois de qualité (Vierzon-Bonaigle, Chœurs, Meillant, etc...), mais leurs ressources sont faibles et près d'être épuisées.

Au total, l'ensemble du groupe Allier, Cher, Indre, ne fournira guère, au cours des 50 ans à̀ venir, qu'un volume de $\mathrm{I}_{5}$ à $20000 \mathrm{~m}^{3}$ de chênes de placage, dont les vestiges des vieux peuplements de Tronça1s représentent l'essentiel. A raison d'un millier de mètres cubes par an, les ressources seront épuisées en moins de 20 ans.

\section{2) Groupe Loir-et-Cher, Indre-et-Loire.}

Les forêts de Blois, Boulogne, Loches, etc..., doivent, à coup sûr, être comprises dans les massifs producteurs de chêne de tranchage. Pour l'instant, les ressources y sont faibles et ne représentent guère plus d'une centaine de mètres cubes de grumes de placage à récolter annnuellement.

\section{3) Groupe Orne et Sarthe.}

Il renferme un ensemble de très belles forêts de chêne: Bellène, Réno-Valdieu, Bourse, Bellebranche, Bercé surtout dont les bois ont toujours été recherchés.

Mais les ressources sont épuisées à Bellème et Bourse. Il ne reste plus à Réno-Valdieu que quelques centaines de mètres cubes de bois de tranchage qui seront exploités en coupes définitives avant Io ans. Bellebranche ne représente encore qu'un canton de jeunes bois.

Seule la forêt de Bercé offre des ressources appréciables et à peu près constantes pour les 50 années à venir, grâce à une bonne ré- 
partition des classes d'âges. Le volume de chênes de tranchage à y récolter chaque année doit avoisiner I $600 \mathrm{~m}^{3}$.

En résumé, les trois groupes de forêts spécialisées du Centre et de l'Ouest présentent une possibilité réduite désormais à $2700 \mathrm{~m}^{3}$ environ.

Les ressources qu'il est possible de tirer sporadiquement des autres forêts domaniales dans le Nivernais, l'Aube, la Haute-Marne, la Côte-d'Or, la Franche-Comté et les Basses-Vosges lorraines et alsaciennes, pour ne citer que ces régions, atteignent un chiffre voisin de 12 à $\mathrm{r} 500 \mathrm{~m}^{3}$.

Au total, les forêts domaniales pourront fournir, au cours des 50 années à venir, un volume annuel de gros chênes voisin de 4000 mètres cubes, en ne tenant compte que des chênes d'un diamètre égal ou supérieur à $0,60 \mathrm{~m}$. Ce chiffre peut être doublé si on considère les chênes de moindre grosseur qui sont pris pour le tranchage.

Mais il existe de nombreuses forêts communales dont les taillissous-futaie vieillis fournissent un contingent important de grumes de placage, probablement égal au volume global des forêts domaniales, soit encore $8000 \mathrm{~m}^{3}$. De même, les exploitants savent très bien recruter par petits paquets, voire pièce par pièce, de gros chênes de qualité, dans les coupes particulières, dans les parcs ou les haies.

$\mathrm{La}$ consommation des usines françaises avoisinant $30000 \mathrm{~m}^{3}$, il faut admettre que ce volume est recruté par parts à peu près égales chez les particuliers et dans les forêts soumises.

\section{Le Bilan EURopéen}

Que reste-t-il à la disposition du commerce européen des chênes de tranchage?

Nous n'avons certes pas énuméré tous les pays susceptibles d'approvisionner le marché. En dehors de l'Allemagne et de la Yougoslavie, il est certain que l'Autriche, la Roumanie, la Belgique, la Tchécoslovaquie et la Pologne possédaient des forêts de chênes fournissant des qualités placages.

En I935, KRAHL-URBAN estimait que l'Autriche pouvait fournir un millier de mètres cubes de ces bois; en I938, Schreiter chiffrait à $6000 \mathrm{~m}^{3}$ le volume de chênes de placage qui pouvait être trouvé dans les forêts de Pologne; pour la Roumanie, nous pouvons nous référer aux descriptions données par SBuRlan des bois de chênes rouvres " à texture faible, à grain fin, à croissance lente, à tige bien élaguée et de belle forme, de belle couleur ", utilisée pour la fabrication des meubles et la tonnellerie, et en induire qu'il s'agit de chênes répondant aux spécifications des bois à placage, s'ils ont les dimensions suffisantes. 
Mais en ce qui concerne la situation présente de ce pays, nous sommes réduits à des hypothèses: si ces chênes sont encore sur pied, quel volume annuel peut être mis sur le marché, et pour quel pays utilisateur?

Limitons-nous, jusqu'à plus ample informé, aux ressources de la France et de l'Allemagne, soit en chiffres ronds 30 à $35000 \mathrm{~m}^{3}$ pour une consommation sensiblement double. Et voici le problème vraiment posé : les besoins en chêne de placage sont à peu près couverts en France, à condition de ne pas être trop exigeant sur la qualité des bois utilisés; pour l'Allemagne, l'industrie du bois joit s'approvisionner à l'extérieur pour les 8/1o de ses besoins; les autres pays utilisateurs (Suisse, Pays-Bas, Grande-Bretagne) n'cnt pas de ressources propres.

La Yougoslavie possède encore des forêts productrices de gros chênes, mais son industrie du placage absorbe de Io à $12000 \mathrm{~m}^{3}$ de grumes de chêne par an. Il ne reste probablement pas un gros volume disponible pour l'exportation.

Or, si nous nous reportons aux statistiques de I938, les feuiliets de chêne de placage exportés par la Yougoslavie, en année moyenne, représentaient 2500 tonnes, qui étaient absorbées:

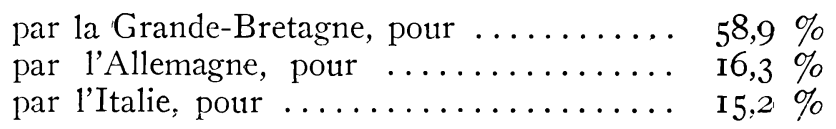

Au total, ce tonnage corresporidait à la mise en œuvre de $8000 \mathrm{~m}^{3}$ de chênes de placage. L'augmentation de consommation intérieure et la diminution des ressources en gros chênes doivent jouer simultanénent pour modifier les tendances du marché du Sud-Est européen.

\section{Conclusion}

La situation présente des possibilités européennes en bois de chêne de placage est inquiétante. La France en profite, qui dispose encore, au milieu d'autres pays qui en sont privés, de ressources en gros chênes qui couvrent les besoins de son industrie et lui permettent, en outre, quelques exportations avantageuses vers la Suisse, l'Allemagne, la Grande-Bretagne et les Pays-Bas.

La France est le pays des chênes à feuilles caduques $(36,5 \%$ de la surface totale occupée par les différentes essences). La proportion des peuplements traités en taillis-sous-futaie a sensiblement diminué, depuis I9I2, en raison des conversions poursuivies de diverses manières, et la surface des peuplements provenant de ces conversions, et acquis à la futaie, est appréciable.

Si les forestiers du $\mathrm{xx}^{\mathrm{e}}$ siècle déplorent que ce régime hybride ait subsisté si longtemps, ils doivent du moins reconnaitre que le chêne a été maintenu, en partie, grâce à lui. Et le volume des ré- 
serves sur pied est assez important pour permettre à l'économie nationale de s'y approvisionner largement en bois de sciages, de charpente et mème de fente.

Or, les méthodes suivies par un grand nombre de propriétaires, à l'exemple de l'Administration forestière, ont occasionné un notable enrichissement de la réserve et la constitution de peuplements assez fermés, où les fûts se sont améliorés. C'est dans certains de ces peuplements, en fin ou en voie de conversion, que devraient être recherchées des billes de qualité moyenne, propres cependant à la fabrication des contreplaqués.

Pour ceux qui ont à organiser la production forestière en France, pour les 50 ans à venir, trois tâches s'imposent, qui ont été déjà énoncées au Congrès international pour l'utilisation du Bois à $\mathrm{Pa}$ ris, en septembre I946:

I) Etablissement d'un inventaire exact des volumes disponibles dans les forêts dont la qualité des bois est reconnue.

2) Recherche de ressources nouvelles dans les massifs non spécialisés.

3) Fixation des possibilités en échelonnant les réalisation des richesses actuelles.

L'amélioration de la situation présente ne peut résulter d'efforts dispersés. Les forestiers qui ont à gérer des forêts de chênes ne doivent pas se considérer comme enfermés dans les limites d'une circonscription. C'est par une mise en commun de ressources, trop restreintes pour créer un marché, que peut être réorgarisée la production.

Et notis pensons que les doctrines d'aménagement par forêt peuvent être revisées lorsqu'il s'agit de tirer le meilleur parti d'tne richesse vraiment nationale.

P. Silvy-Leligois. 


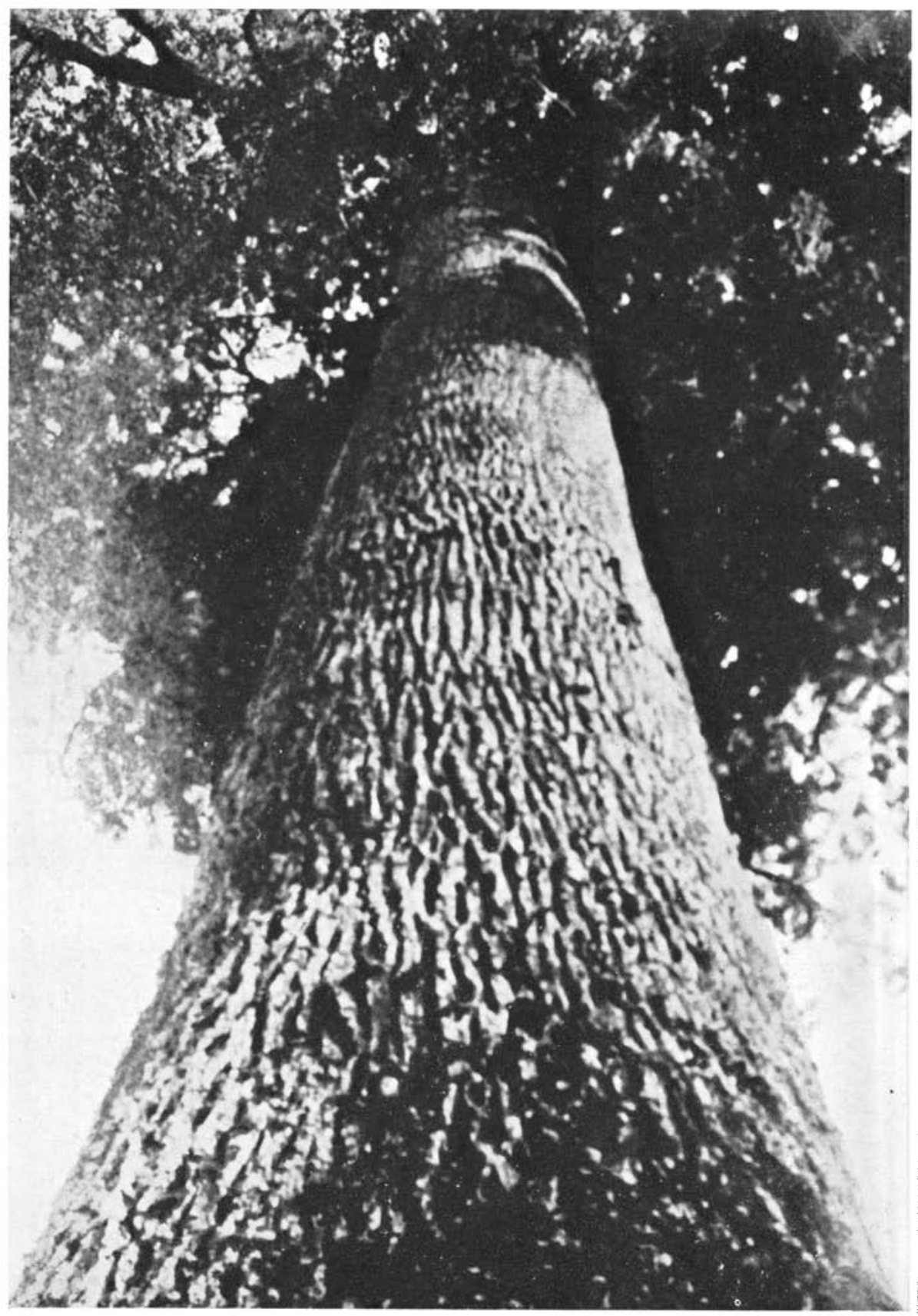

France

Forêt de Tronçais. Chêne de Buffévent

Fût de chêne rouvre de 260 à 280 ans.

Diam.: I,56 m; hauteur du fût: $18 \mathrm{~m}$; hatuteur totale: $34 \mathrm{~m}$.

(Cliché François Kollar.)

Extrait de La France travaille.

Reproduit avec l'aimable autorisation de Horizons de France. 
LA PRODUCTION DES CHÊNES DE TRANCHAGE

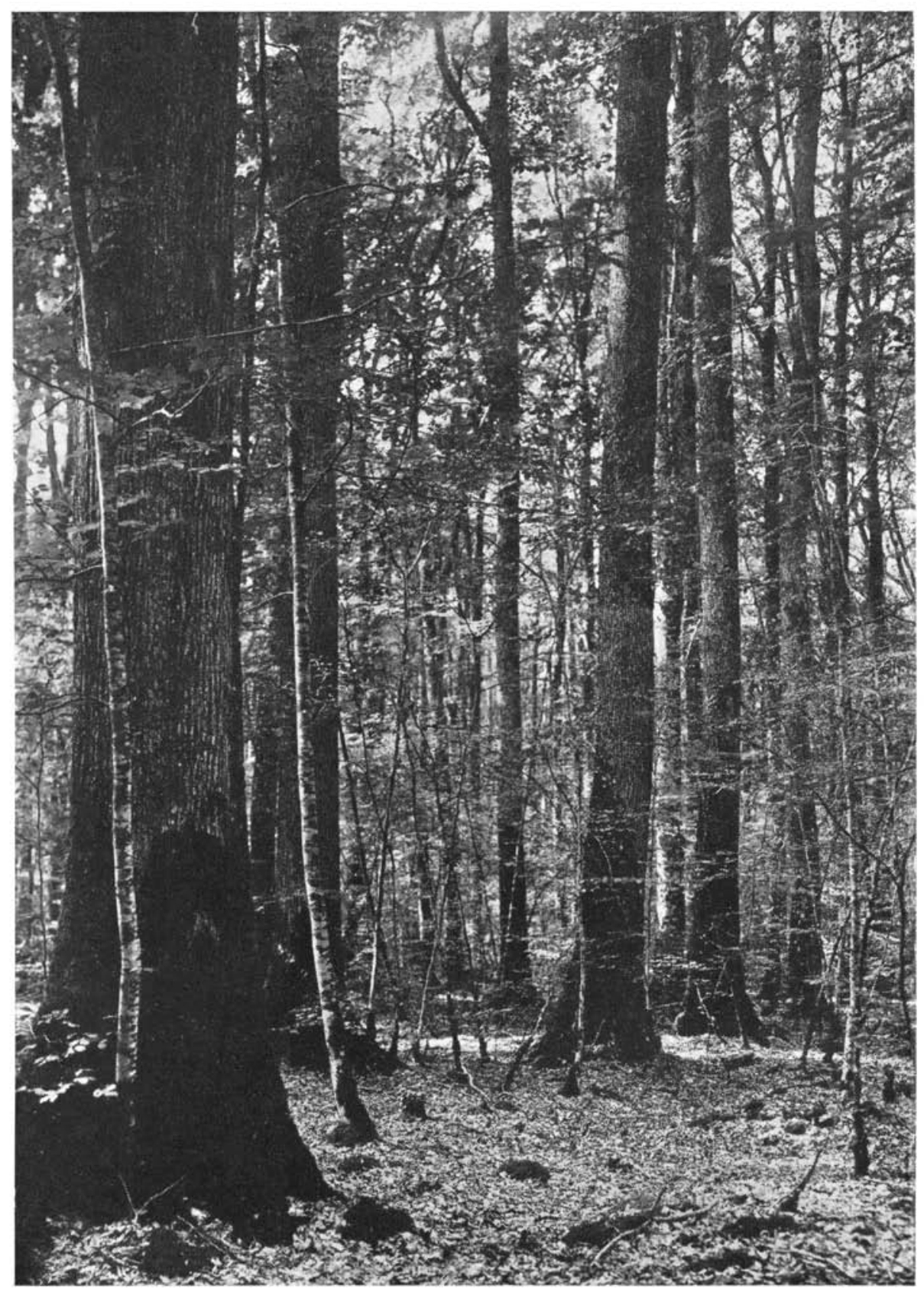

Forêt de Bercé. Canton des Pressenteries.

Vieille futaie de Chêne rouvre (220 ans) avec sous-étage de Hêtre

(Cliché Thiollier.) 